\title{
RMetS
}

Royal Meteorological Society

\section{Absolute or convective instability in the equatorial Pacific and implications for ENSO}

\author{
Sulian Thual, ${ }^{\mathrm{a}}$ Olivier Thual ${ }^{\mathrm{b}, \mathrm{c}}$ and Boris Dewitte ${ }^{\mathrm{a}}$ \\ ${ }^{a}$ Laboratoire d'Etude en Géophysique et Océanographie Spatiale, Toulouse, France \\ ${ }^{\mathrm{b}}$ Université de Toulouse; INPT, UPS; IMFT, Toulouse, France \\ ${ }^{\mathrm{c}} \mathrm{CNRS}$; IMFT, Toulouse, France
}

*Correspondence to: O. Thual, IMFT, Allée C. Soula, Toulouse 31400, France. E-mail: thual@imft.fr

The El Niño-Southern Oscillation (ENSO) is driven by ocean-atmosphere interactions in the equatorial Pacific, and this variability is often attributed to coupled modes that are evidenced by the temporal stability analysis of anomaly models. Here, the further diagnostic of absolute/convective instability is considered, which assesses whether small perturbations localized in space and time (e.g. random wind disturbances) lead to instabilities that develop in-place or propagate away from the perturbed region. It is shown that boundary conditions play a secondary role for this approach and that the development of large-scale wave packets in the equatorial Pacific basin is possible, as in the case of an infinite domain. As an illustration, two simple coupled models are diagnosed that rely either on thermocline processes or zonal advective processes. The model with thermocline processes is 'absolutely unstable' and therefore develops intrinsic oscillations, while the model with zonal advective processes is 'convectively unstable' and therefore acts as a noise amplifier. The identification of the two instability regimes may characterize different ENSO formation mechanisms as a response to random wind disturbances. For the absolutely unstable regime, a standing ENSO-like oscillation can develop in the equatorial Pacific without involving boundary reflections, while for the convectively unstable regime boundary reflections are essential. Copyright (c) 2012 Royal Meteorological Society

Key Words: ENSO; equatorial Pacific; interannual variability; absolute instability; wave packets; conceptual models

Received 5 February 2012; Revised 9 May 2012; Accepted 23 May 2012; Published online in Wiley Online Library 19 July 2012

Citation: Thual S, Thual O, Dewitte B. 2013. Absolute or convective instability in the equatorial Pacific and implications for ENSO. Q. J. R. Meteorol. Soc. 139: 600-606. DOI:10.1002/qj.1988

\section{Introduction}

A hierarchy of models of increasing complexity simulate the interannual variability of the equatorial Pacific with a particular focus on the forecast of El Niño/La Niña events and, more generally, explain the main features of the El Niño-Southern Oscillation (hereafter ENSO). In those models, the development of anomalies is mainly attributed to interactions between the ocean and the atmosphere. The studies of temporal instabilities exhibited by those models have led to the emergence of the theory of coupled modes (Neelin et al., 1998). It also provides a fruitful view for the interpretation of observations and coupled general circulation models.

The temporal stability analysis of models has allowed the identification of various types of coupled modes of the equatorial Pacific. They are usually classified depending on the physical processes involved. A large number of studies distinguish two families of coupled modes either based on thermocline processes or zonal advective processes in the ocean (Hirst, 1986; Bejarano and Jin, 2008; Thual et al., 2011). An and Jin (2001) and Fedorov and Philander 
(2001) further suggest that the relative strength of those two processes can lead to different regimes of the ENSO in terms of amplitude, frequency and propagation. Other relevant coupled modes have been evidenced that rely solely on the interaction between the atmosphere and the oceanic mixed layer (Wang and Xie, 1998; Dommenget, 2010). Asymptotic limits for the adjustment time of each of the coupled system components are also important. Neelin (1991) distinguishes the coupled modes where the adjustment time of sea-surface temperature (SST) is fast compared to the adjustment time of the ocean dynamics (the fast-SST regime) and inversely (the fast-wave regime). Furthermore, within this line of studies, it is usually assumed that a 'fast atmosphere' adjusts immediately to SST. Although the reflections at the eastern and western boundaries are usually considered in coupled models, some noticeable studies identify coupled modes without boundary conditions (Hirst, 1986; Wang and Weisberg, 1996; Weisberg and Wang, 1997; Wang and Xie, 1998; Pontaud and Thual, 1995, 1998).

A further approach to temporal stability analysis is the diagnostic of absolute/convective instability. So far, this approach has not been considered for the identification of coupled modes in the equatorial Pacific. The diagnostic of absolute/convective instability originates from plasma physics (Briggs, 1964) and has been further developed in fluid mechanics. A review of the method is given in Huerre and Monkewitz (1990), a discussion on its practical implementation in Suslov (2006), and a few applications in geophysical fluid dynamics stability problems are listed in Pedlosky (2012). The diagnostic assesses whether small perturbations localized in space and time lead to instabilities that develop in-place or propagate away from the perturbed region. Absolutely unstable systems develop intrinsic oscillations in the perturbed region: they act as 'oscillators' with intrinsic dynamics. Convectively unstable systems amplify but disperse the external perturbations: they act as 'noise amplifiers' with extrinsic dynamics.

In most ENSO theories, boundary reflections are essential for the system to oscillate, as they provide the necessary negative feedback for the transition from a warm (El Niño) phase to a cold (La Niña) phase and inversely (Suarez and Schopf, 1988; Jin, 1997; Picaut et al., 1997). Those theories consider coupled modes that are solutions of a system with prescribed boundary conditions. Absolute or convective instabilities are of a different nature, although they are not incompatible with the presence of boundary conditions. They are described in terms of wave packets, which result from the superposition of a continuum of modes that are solutions of a linear system in an infinite domain. When further considering boundary conditions, the reflected components simply superpose in a linear framework. The diagnostic of absolute/convective instability depicts whether the stationary component decays or develops into a standing oscillation, independently of the reflected components.

The diagnostic may characterize different ENSO formation mechanisms as a response to random disturbances, e.g. westerly winds bursts (Harrison and Vecchi, 1997). Different scenarios can be expected depending on the stability of the equatorial Pacific system (Philander and Fedorov, 2003; Kleeman, 2008; Kessler, 2002). If the system is strongly damped, the ENSO has to be only a direct response to random wind disturbances, but this hypothesis is unlikely. If the system is unstable enough, it may be self-regulated by internal nonlinear processes, such as overlapping resonance with the seasonal cycle (Jin et al., 1994) or mean state rectification by the heat budget asymmetry (Timmermann et al., 2003). The diagnostic presented here applies in the intermediate case of a slightly unstable system. As stressed by Neelin et al. (1998), for such a system the ENSO is weakly nonlinear, in the sense that dominant spatial and temporal scales of variability are determined by the leading coupled mode(s) issued from linear stability. Random wind disturbances are not necessary to maintain the variability but still act as a trigger of specific events, through small perturbations that are amplified by the coupled system in the form of wave packets. As a response to random wind disturbances, in the absolutely unstable regime an ENSO cycle can develop as a standing oscillation, while in the convectively unstable regime an ENSO cycle may still develop but only if boundary reflections are involved.

In this article, the approach is illustrated by diagnosing two simple coupled models of the equatorial Pacific that differ in the thermodynamic processes considered. We aim at evaluating, among those processes, those that lead either to an absolute or to a convective regime. The models are presented in section 2 . Section 3 is dedicated to a temporal stability analysis of the models as a consistency check with previous studies. In section 4 we diagnose the models in terms of absolute/convective instability. Section 5 is a discussion, followed by concluding remarks.

\section{Coupled system}

We consider a shallow-water model for the ocean in the long-wave approximation and in an infinite domain. This system is projected on the meridional basis of 'oceanic' Hermite functions (with oceanic Rossby radius $\sqrt{c_{o} / \beta}$ ) and truncated at the Kelvin and first Rossby equatorial wave components, $K(x, t)$ and $R(x, t)$. Those depend on longitude and time through

$$
\begin{aligned}
& \left(\partial_{t}+\varepsilon_{o}\right) K+c_{o} \partial_{x} K=P_{K}\left(\tau_{x}\right), \\
& \left(\partial_{t}+\varepsilon_{o}\right) R-\frac{c_{o}}{3} \partial_{x} R=P_{R}\left(\tau_{x}\right),
\end{aligned}
$$

where $\varepsilon_{o}$ is the dynamical damping, $c_{o}$ is the phase speed of the oceanic Kelvin waves, $\tau_{x}(x, y, t)$ is the field of zonal wind stress anomalies $\left(\mathrm{N} \mathrm{m}^{-2}\right)$ and $P_{K}$ and $P_{R}$ are projection operators on the oceanic Hermite functions. The parameter values are those from Thual et al. (2011) when considering only the contribution from the first baroclinic mode of the ocean. The approach is similar for the atmosphere. We consider a Gill-type shallow-water model in the long-wave approximation (Gill, 1980). The system is projected on the meridional basis of 'atmospheric' Hermite functions (with atmospheric Rossby radius $\sqrt{c_{a} / \beta}$ ) and truncated at the Kelvin and first Rossby equatorial wave components, $k(x, t)$ and $r(x, t)$. Those depend on longitude and time through

$$
\begin{aligned}
& \varepsilon_{a} k+c_{a} \partial_{x} k=P_{k}(T), \\
& \varepsilon_{a} r-\frac{c_{a}}{3} \partial_{x} r=P_{r}(T),
\end{aligned}
$$

where $\varepsilon_{a}$ is the atmospheric dynamical damping, $c_{a}$ is the phase speed of the atmospheric Kelvin waves, $T(x, y, t)$ is the field of SST anomalies and $P_{k}$ and $P_{r}$ are projection operators on the atmospheric Hermite functions. The parameter values 
are those from Hirst (1986). The adjustment time of the atmosphere (a few days) is neglected compared to the one of the ocean (a few months). Finally, we consider a linearized mixed-layer thermodynamic model for SST anomalies $T$ and retain only the dominant thermodynamic processes, namely the feedbacks due to damping, zonal advection and thermocline depth anomalies:

$$
\left(\partial_{t}+\varepsilon_{T}\right) T=f_{H} H+f_{U} U
$$

where $\varepsilon_{T}$ is the thermodynamic damping and $f_{H}$ and $f_{U}$ are constant coefficients. Model parameters are derived from Thual et al. (2011), where further considering constant and representative values of the thermocline feedback efficiency factor $\left(\gamma=0.1^{\circ} \mathrm{C} \mathrm{m}^{-1}\right)$ and the mean SST zonal gradient $\left(\partial_{x} T=-10^{\circ} \mathrm{C} / L\right.$, where $L$ is the basin length of $17000 \mathrm{~km}) . H(x, y, t)$ and $U(x, y, t)$ are fields of thermocline depth anomalies $(\mathrm{m})$ and zonal current anomalies $\left(\mathrm{m} \mathrm{s}^{-1}\right)$ respectively, which can be reconstructed from $K$ and $R$ using the oceanic Hermite functions. Similarly, $\tau_{x}(x, y, t)$ can be reconstructed from $k$ and $r$ using the atmospheric Hermite functions if assuming, as in Hirst (1986), that wind stress is proportional to wind speed. In order to eliminate the variables $\tau_{x}, T, H$ and $U$ we project and combine the oceanic, atmospheric and thermodynamic equations. We obtain a linear system of four variables $K, R, k, r$ that depend on longitude and time:

$$
\begin{aligned}
& \left(\partial_{t}+\varepsilon_{o}\right) K+c_{o} \partial_{x} K=M_{K k} k+M_{K r} r, \\
& \left(\partial_{t}+\varepsilon_{o}\right) R-\frac{c_{o}}{3} \partial_{x} R=M_{R k} k+M_{R r} r, \\
& \left(\partial_{t}+\varepsilon_{T}\right)\left(k+\frac{c_{a}}{\varepsilon_{a}} \partial_{x} k\right)=M_{k K} K+M_{k R} R, \\
& \left(\partial_{t}+\varepsilon_{T}\right)\left(r-\frac{c_{a}}{3 \varepsilon_{a}} \partial_{x} r\right)=M_{r K} K+M_{r R} R,
\end{aligned}
$$

where $M_{K k}, M_{K r}, M_{R k}, M_{R r}, M_{k K}, M_{k R}, M_{r K}$ and $M_{r R}$ are constant coefficients. Interestingly, the phase speed resulting from the combined atmosphere and thermodynamics $\varepsilon_{T} c_{a} / \varepsilon_{a} \sim 2 \mathrm{~m} \mathrm{~s}^{-1}$ is close to the phase speed of the ocean model $c_{o} \sim 3 \mathrm{~m} \mathrm{~s}^{-1}$. The resulting damping $\varepsilon_{T} \sim$ $(35 \text { day })^{-1}$ is, however, much stronger than for the ocean model $\varepsilon_{o}=(2.5 \text { year })^{-1}$.

We consider two variants of this coupled system, which differ by the thermodynamic processes involved. The distinction between thermocline and zonal advective processes has already been adopted in various studies of the equatorial Pacific coupled modes, as it leads to two families of coupled modes that have different properties and that are both relevant for interpreting the interannual variability. We consider a first model (hereafter $\mathrm{H}$-model) where only thermocline processes are considered $\left(f_{U}=0\right)$, and a second model (hereafter U-model) where only zonal advective processes are considered $\left(f_{H}=0\right)$. Note that we also implemented a third model where both processes are considered, which is not documented here since the main conclusions are similar to those of the H-model. For numerical applications, $\varepsilon_{o}=(30 \mathrm{month})^{-1}, c_{o}=2.8 \mathrm{~m} \mathrm{~s}^{-1}$, $\varepsilon_{a}=\left(2.3 \mathrm{day}^{-1}, c_{a}=30 \mathrm{~m} \mathrm{~s}^{-1}\right.$, and $\varepsilon_{T}=(35.7 \mathrm{day})^{-1}$. In both models, $M_{K k}=-6.0, M_{K r}=8.4, M_{R k}=2.3$, and $M_{R r}=-3.9$ (numerical values are given in year ${ }^{-1}$ ). In the H-model $M_{k K}=32.1, M_{k R}=45.3, M_{r K}=12.5$, and $M_{r R}=$ 21.1. In the U-model $M_{k K}=16.8, M_{k R}=-9.9, M_{r K}=6.6$ and $M_{r R}=-2.1$.

\section{Temporal stability analysis}

We search for one-dimensional vector fields $\boldsymbol{X}(x, t)=$ $[K, R, k, r]$ that are solutions of the coupled system of the previous section. Since the system is real, linear and homogeneous in an infinite domain, one can consider complex solutions of the form $\boldsymbol{X}=\boldsymbol{X}_{0} \exp [i(k x-\omega t)]$, where $k=k_{r}+i k_{i}$ and $\omega=\omega_{r}+i \omega_{i}$ are respectively the complex wave number and frequency ( $i$ is the imaginary number). Non-trivial solutions are obtained when a couple $(k, \omega)$ satisfies the generalized dispersion relation $D(k, \omega)=0$, which is obtained through the transformations $\partial_{x}=i k$ and $\partial_{t}=-i \omega$ followed by a kernel analysis of the resulting algebraic system. The temporal stability consists, for a determined real wave number $k_{r}$, in evaluating the complex roots $\omega$ of the dispersion relation $D\left(k_{r}, \omega\right)=0$. As a preliminary step to the diagnostic of absolute/convective instability, we comment here on the temporal stability analysis of each model as a consistency check with previous studies.

The temporal stability of the H-model is shown in Figure 1. At large wave number, the modes are identical to the uncoupled case, with an 'oceanic Kelvin coupled mode' (yellow) and an 'oceanic Rossby coupled mode' (green) that propagate at speed $c_{o}$ and $-c_{o} / 3$ respectively $\left(c_{o} \sim 3 \mathrm{~m} \mathrm{~s}^{-1}\right)$ and that are slightly damped $\left(\omega_{i} \sim-1\right.$ year $^{-1}$ ), as well as two 'atmospheric coupled modes' (cyan and blue) that do not propagate and that are strongly damped $\left(\omega_{i} \sim-10\right.$ year $\left.^{-1}\right)$. For a decreasing wave number, one of the atmospheric coupled modes (blue) eventually becomes unstable. Neelin (1991) named this type of unstable mode the 'slow SST mode', where the ocean dynamics adjustment is fast compared to the SST adjustment. This slow SST mode propagates eastward for $k_{r} \sim 0.2(1000 \mathrm{~km})^{-1}$ and westward for $k_{r}<0.1(1000 \mathrm{~km})^{-1}$, and has maximal amplitude on the atmospheric Kelvin wave (not shown). The temporal stability of the U-model is shown in Figure 2. In this model the oceanic Kelvin coupled mode (yellow) and the second atmospheric coupled mode (cyan) are similar to those of the H-model. However, the slow SST mode (blue) remains damped and the oceanic Rossby coupled mode (green) is slightly unstable. This oceanic Rossby coupled mode propagates westward as in the uncoupled case, and
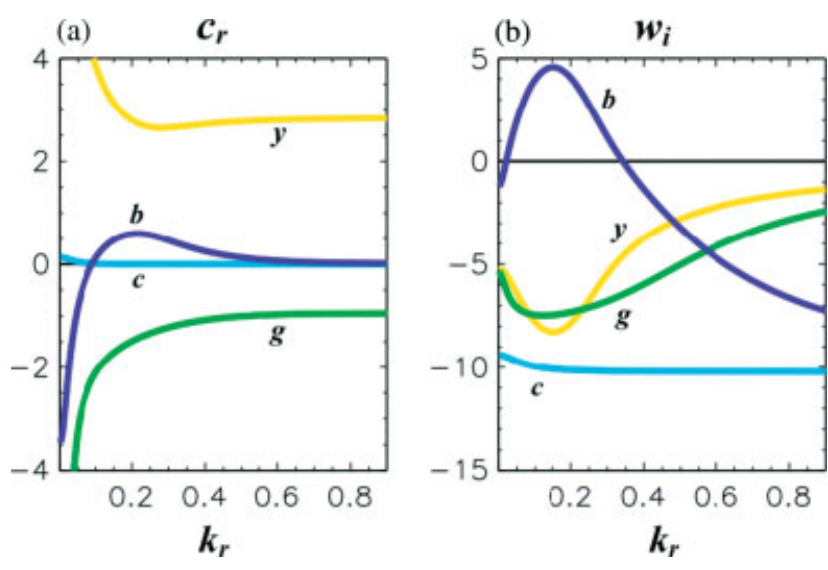

Figure 1. Temporal stability of the H-model. Results are shown for the four mode solution of the dispersion relation, which are identified by colours (yellow, green, cyan, blue). (a) Phase speed $c_{r}=\omega_{r} / k_{r}\left(\mathrm{~m} \mathrm{~s}^{-1}\right)$ as a function of the real wave number $k_{r}$ (unit is $(1000 \mathrm{~km})^{-1}$ ). For comparison, the equatorial Pacific length is $17000 \mathrm{~km}$, which corresponds to a wave number around $0.4(1000 \mathrm{~km})^{-1}$. (b) Growth rate $\omega_{i}\left(\right.$ year $\left.^{-1}\right)$ as a function of $k_{r}$. This figure is available in colour online at wileyonlinelibrary.com/journal/qj 

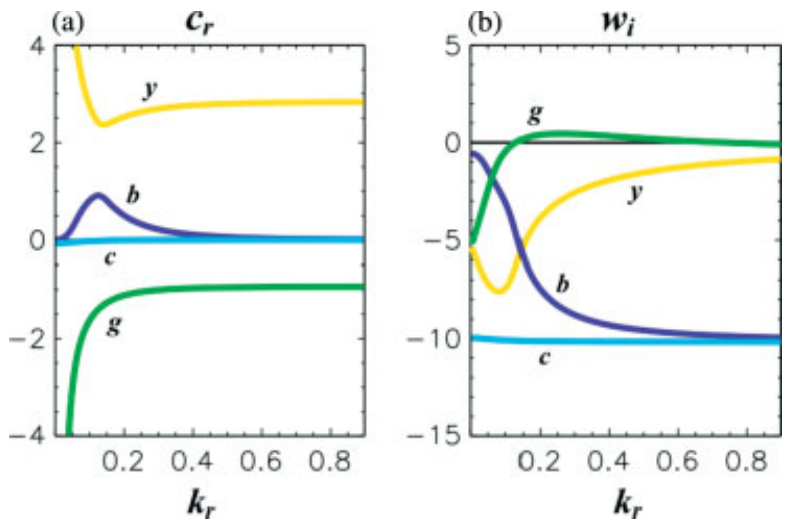

Figure 2. Same as Figure 1 but for the U-model. This figure is available in colour online at wileyonlinelibrary.com/journal/qj

has maximal amplitude on the oceanic Rossby wave (not shown). As in Hirst (1986), we have tested the case with an opposite mean SST gradient $\left(f_{U}<0\right)$, which leads to an opposite direction of propagation, i.e. a slightly unstable oceanic Kelvin coupled mode (not shown). The results agree overall with Hirst (1986, their Figures 7 and 13), Wang and Weisberg (1996, their Figure 1) and Wang and Xie (1998, their Figure 9).

The results from Hirst (1986, their Figure 20), however, exhibit great sensitivity to the number of Hermite functions retained for the meridional truncation. As a further test, we recomputed the temporal stability of the $\mathrm{H}$-model and U-model when increasing the number of meridional components in both the ocean and atmosphere (only odd, i.e. symmetrical Rossby components, were considered). We find that this only slightly modifies the behaviour of the most unstable modes (not shown). We believe that this interesting property of our system is due to the use of an adapted Hermite basis for the atmosphere (with atmospheric Rossby radius), different from the Hermite basis used for the ocean (with oceanic Rossby radius). In Hirst (1986), the Hermite basis of the ocean is used to project the entire system, therefore a large number of meridional modes are necessary to represent the larger meridional scale of the atmosphere.

In the infinite domain considered here, the above temporal stability analysis can be used to describe wave packets made of the superposition of a continuum of modes with wave numbers close to a given real value $k_{r}$. Such wave packets propagate at group velocity $\partial \omega_{r} / \partial k_{r}$. One can compute the growth rate $\omega_{i}$ of the wave packets propagating at zero group velocity and document their temporal growth or decay at a given location. However, such a consideration must be extended with the study of 'generalized wave packets' built with a continuum of complex wave numbers, i.e. waves with both a wave number $k_{r}$ and a spatial growth rate $-k_{i}$. Indeed, the most unstable of the generalized wave packets with zero group velocity will provide the local response of the system to a localized perturbation. This analysis is formalized through the diagnostic of absolute/convective instability described and applied to our models in the next section.

\section{Absolute or convective instability}

We introduce the diagnostic of absolute/convective instability following Huerre and Monkewitz (1990) and apply it to the models described in the previous sections. Such a diagnostic is relevant only for systems that are linearly unstable. This is the case for the H-model and the U-model, where the temporal analysis evidences the unstable slow SST mode and the slightly unstable oceanic Rossby coupled mode respectively (see section 3 ). For such a system, we aim at evaluating the system response $G(x, t)$ to a pulse in space and time, which is a solution of

$$
D\left(-i \partial_{x}, i \partial_{t}\right) G(x, t)=\delta(x) \delta(t)
$$

where $D(k, \omega)$ is the dispersion relation of the system, $i$ is the complex number and $\delta$ is the Dirac function. For this problem, $G$ is the Green function, which can be computed through integration in the complex space as

$$
G(x, t)=\frac{1}{(2 \pi)^{2}} \iint \frac{\exp (i(k x-\omega t))}{D(\omega, k)} \mathrm{d} \omega \mathrm{d} k,
$$

where $\omega$ and $k$ are complex. We suppose for simplicity that the dispersion relation admits a single root $\omega(k)$ (the demonstration can be extended to the case of multiple roots), and we use the residual method to integrate over the path $\omega(k)$ :

$$
G(x, t)=\frac{-i}{2 \pi} \int_{-\infty}^{+\infty} \frac{\exp (i(k x-\omega t))}{\partial_{\omega} D(\omega, k)} \mathrm{d} k,
$$

where $t \geq 0$. We consider solutions over a ray path at constant (and real) velocity $V\left(\mathrm{~m} \mathrm{~s}^{-1}\right)$, verifying $x=V t$. For large $t$, we can approximate asymptotically the integral with the steepest-descent method, provided we find a saddle point of the complex function $\rho(k)=i[k V-\omega(k)]$, i.e. a complex root $k_{0}$ of $(\mathrm{d} \rho / \mathrm{d} k)\left(k_{0}\right)=0$. If such a root exists, the asymptotic expansion for large $t$ reads

$$
G(V t, t) \sim-\exp (i \pi / 4)\left(2 \pi t d_{k}^{2} \omega\right)^{-1 / 2} \frac{\exp (\rho)}{\partial_{\omega} D},
$$

where the right member is taken at $k_{0}=k_{0 r}+i k_{0 i}$ and at the corresponding $\omega_{0}=\omega_{0 r}+i \omega_{0 i}$. This shows that the response is a 'generalized wave packet' of wavelength $2 \pi / k_{0 r}$ and spatial growth rate $-k_{0 i}$ that travels at speed $V=(\mathrm{d} \omega / \mathrm{d} k)\left(k_{0}\right)$ with a temporal growth rate equal to $\operatorname{Re}\left[\rho\left(k_{0}\right)\right]$. If multiple roots exist, the behaviour is dominated by the most unstable root.

Such behaviour is overlooked in particular for $V=0$, to distinguish between the absolutely unstable and convectively unstable regimes. If for $V=0$ the temporal growth rate $\operatorname{Re}\left[\rho\left(k_{0}\right)\right]$ is positive (which reads $\omega_{0 i}>0$ ), then the system is said to be absolutely unstable, meaning that an unstable generalized wave packet develops in the perturbed region. If for $V=0$ the temporal growth rate is negative, it is said to be convectively unstable. Such a system has to be at least unstable for one non-null value of $V$, because it is nevertheless linearly unstable according to temporal stability. Supposing that such an unstable generalized wave packet develops, then, in the meantime, it propagates away at non-null speed $V$ from the perturbed region and is evacuated at a large time-scale. Therefore, in the perturbed region the signal is dispersed and decays, similarly to the linearly stable regime.

In general, searching the saddle points of the system can require an important practical implementation, especially if 

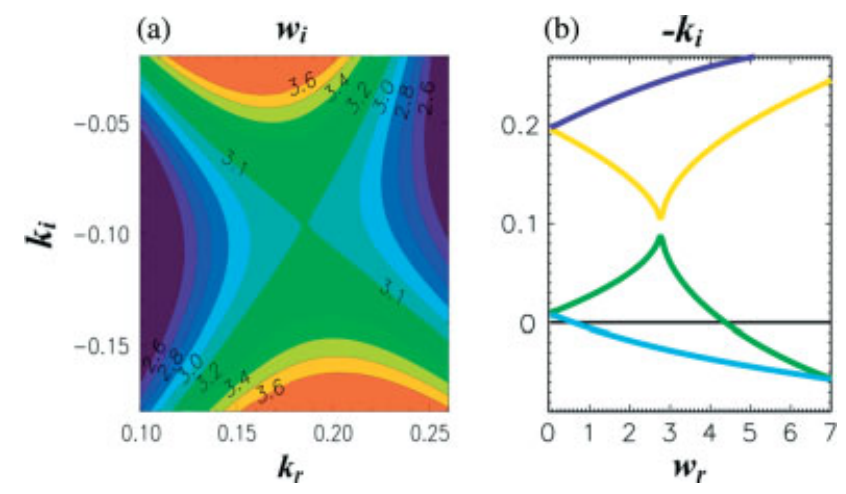

Figure 3. Saddle point detection for the H-model. (a) Imaginary frequency $\omega_{i}\left(\right.$ year $\left.^{-1}\right)$ as a function of the real wave number $k_{r}$ (X-axis, unit is $\left.(1000 \mathrm{~km})^{-1}\right)$ and the imaginary wave number $k_{i}$ ( $Y$-axis, unit is $\left.(1000 \mathrm{~km})^{-1}\right)$. The saddle point is found at the intersection of the isocontours for $\omega_{0 i} \sim 3.1$ year $^{-1}$. (b) Imaginary wave number $-k_{i}$ (unit is $\left.(1000 \mathrm{~km})^{-1}\right)$ as a function of the real frequency $\omega_{r}\left(\right.$ year $\left.^{-1}\right)$ and for $\omega_{i}=$ $\omega_{0 i}$. The saddle point is found at the intersection of two roots $k(\omega)$. This figure is available in colour online at wileyonlinelibrary.com/journal/qj

automation is required (Suslov, 2006). The coupled system considered here, with four variables, is, however, rather simple thanks to the system truncation at the first meridional Hermite functions. We use a graphical method for the detection of saddle points, which is illustrated in Figure 3 for the H-model. At first, the saddle points for $V=0$ are detected in the wave number complex space $\left(k_{r}, k_{i}\right)$ at the intersections of the isocontours of the imaginary frequency $\omega_{i}$ (Figure 3(a)). At least one saddle point must be detected. The system is absolutely unstable provided that the most unstable saddle point verifies $\omega_{0 i}>0\left(\omega_{0 i}\right.$ is the absolute growth rate). Also one can easily show that $\omega_{0 i}$ is always inferior or equal to the maximal growth rate from the temporal stability. As stressed by Huerre and Monkewitz (1990), for the absolute instability to be assessed the most unstable saddle point must also satisfy the Briggs (1964) criterion: such a saddle point must be at the intersection $\left(\omega=\omega_{0}\right)$ of two roots $k(\omega)$ that originate from the upper and lower parts of the $k$-plan $\left(k_{i}>0\right.$ and $\left.k_{i}<0\right)$ when increasing $\omega_{i}$. We have checked that this was the case (Figure 3(b)).

We have found that the H-model is absolutely unstable, while the U-model is convectively unstable. For the $\mathrm{H}$ model the absolute growth rate is $\omega_{0 i}=3.1$ year $^{-1}$ and the absolute frequency is $\omega_{0 r}=2.8$ year $^{-1}$ (period is $2 \pi / \omega_{0 r} \sim 2.2$ years $)$. This is consistent with contemporary
ENSO characteristics (i.e. a maintained oscillation with a period of 2 to 7 years). The associated saddle point is found at $k_{0 i} \sim-0.1(1000 \mathrm{~km})^{-1}$ and $k_{0 r} \sim 0.2(1000 \mathrm{~km})^{-1}$ (i.e. a wavelength $2 \pi / k_{0 r} \sim 30000 \mathrm{~km}$, which is about twice the equatorial Pacific basin length). The oscillation therefore occurs at a basin scale. For the U-model there are no unstable $\left(\omega_{0 i}>0\right)$ roots, therefore the system is convectively unstable.

As a consistency test, we simulated numerically the impulse response of both models, and the results are illustrated in Figure 4. In good agreement with the diagnostic of absolute/convective instability, for the H-model, which is absolutely unstable, the amplitude of the response grows in-place of the initial perturbation, while for the U-model, which is convectively unstable, the amplitude decays.

We also investigated results as a function of the intensity of the thermodynamic feedback considered (either thermocline or zonal advective feedback). In both models, we found a first transition from the linearly stable regime to the convectively unstable regime when increasing the intensity from zero, followed by a second transition from the convectively unstable regime to the absolutely unstable regime. In the $\mathrm{H}$-model, the first transition occurs at around $14 \%$ of the reference value of $f_{H}$ and the second transition at around $16 \%$. It is therefore unlikely that the $\mathrm{H}$-model sustains a convective regime within such a narrow range of parameter values. In the U-model the first transition occurs at around $50 \%$ of the reference value of $f_{U}$ and the second transition at around $130 \%$. Therefore the U-model is preferentially in the convective regime. When the Umodel is in the absolute regime $\left(f_{U} \sim 130 \%\right.$ to $200 \%$ of its reference value), however, it sustains a high-frequency oscillation $\left(2 \pi / \omega_{0 r} \sim 0.9\right.$ year $)$ that is only slightly unstable $\left(\omega_{0 i}<1.3\right.$ year $\left.^{-1}\right)$.

\section{Discussion}

In this article we have considered a simple coupled system of the equatorial Pacific in an infinite domain, using shallow-water dynamics for both the ocean and atmosphere in the long-wave approximation and truncating at the Kelvin and first-meridional Rossby waves. Two variants of the coupled system were considered, which differ by the thermodynamic processes controlling the variations in SST. At first, the models were diagnosed in terms of temporal stability. In agreement with previous studies,
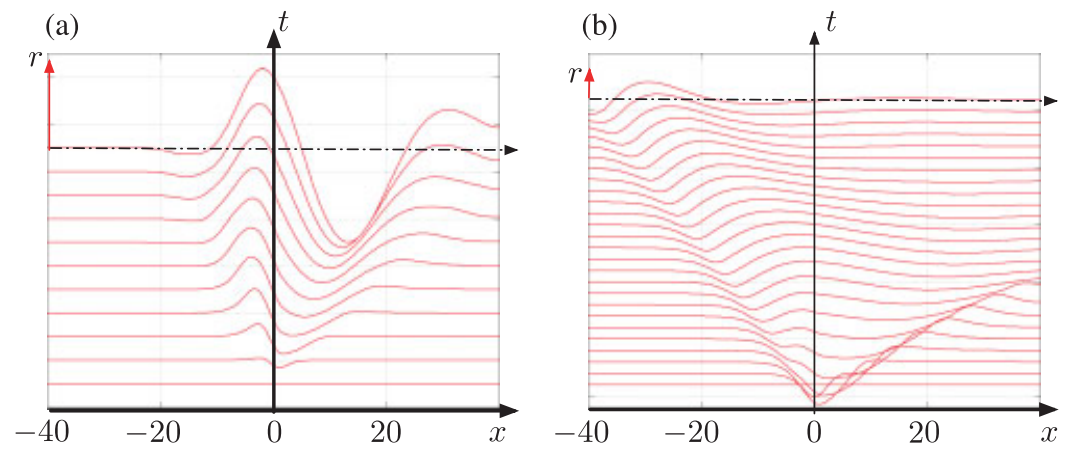

Figure 4. Response to an initial and localized perturbation. The atmospheric Kelvin profile is initialized with a Gaussian shape of $1000 \mathrm{~km}$ root mean square centred at $x=0$. The amplitude of the atmospheric Rossby profile $r(x, t)$ is represented as a function of $x$ (unit is $1000 \mathrm{~km}$ ) as $t$ increases by steps of 20 days. For comparison, the equatorial Pacific basin length is about $17000 \mathrm{~km}$. (a) Absolute instability for the H-model: the amplitude grows in-place of the initial perturbation (time increases up to 200 days). (b) Convective instability for the U-model: the amplitude decays in-place of the initial perturbation (time increases up to 500 days). This figure is available in colour online at wileyonlinelibrary.com/journal/qj 
the model with thermocline processes (H-model) shows an unstable slow SST mode, while the model with zonal advective processes (U-model) shows a slightly unstable oceanic Rossby coupled mode. Then, the models were diagnosed in terms of absolute/convective instability. Within a representative range of parameters values, the $\mathrm{H}$-model is absolutely unstable and the $\mathrm{U}$-model is convectively unstable.

In the H-model, spatial and temporal scales of the absolute instability are consistent with contemporary ENSO characteristics, namely a basin-scale interannual oscillation. This suggests that a standing ENSO-like oscillation can develop in the equatorial Pacific without involving boundary reflections. Here, the ENSO develops on a continuum of coupled modes that can be viewed as 'generalized wave packets', which are considered in an infinite domain and that are not inhibited by the addition of boundary conditions. In most ENSO theories, the dynamics are rather described by coupled modes that are temporal 'basin modes', which are quantified as they satisfy boundary conditions (e.g. as the harmonics of a vibrating string). Temporal basin modes were not considered here, but they can coexist with the generalized wave packets in a linear framework. However, generalized wave packets cannot be constructed as a continuum of temporal basin modes because their asymptotic behaviour for large time is in $\exp (\rho t) / \sqrt{t}$. In the case of an absolutely unstable system, generalized wave packets may be at least as important for ENSO formation as temporal basin modes. In the case of a convectively unstable system, only temporal basin modes may develop an ENSO oscillation, therefore basin parameters (its geometry and reflection coefficients) should be important.

There is an ongoing discussion on whether ENSO is a series of events triggered by random wind disturbances or a self-sustained natural mode of the coupled ocean-atmosphere. The diagnostic presented here, within the assumed hypothesis, may eventually provide material for this discussion. It is assumed here that the interannual variability results from the linear amplification of random wind disturbances, which leads to different ENSO formation mechanisms depending on the regime of the equatorial Pacific system. The regime (absolutely unstable, convectively unstable or linearly stable) may change depending on the mean state, and therefore it would be fundamental to map transitions as a function of the many mean state parameters.

So far, we have considered local modes where the mean state is constant or varies very slowly in space as compared to the wavelength of the problem. The diagnostic of absolute/convective instability may, however, be different when considering various regions as a whole, with different properties. Such a diagnostic for inhomogeneous systems in space requires the concept of global modes (Huerre and Monkewitz, 1990). Since the equatorial Pacific shows an important zonal contrast in its mean state parameters, it would be interesting to consider global modes where this contrast is taken into account. The equatorial Pacific can be schematically divided into the Eastern Pacific (Niño3 region: $150^{\circ} \mathrm{W}-90^{\circ} \mathrm{W}, 5^{\circ} \mathrm{S}-5^{\circ} \mathrm{N}$ ) dominated by thermocline processes and the Central Pacific (Niño4 region: $\left.150^{\circ} \mathrm{E}-150^{\circ} \mathrm{W}, 5^{\circ} \mathrm{S}-5^{\circ} \mathrm{N}\right)$ dominated by zonal advective processes (An and Jin, 2001). According to our results (where only the thermocline process leads to the absolute regime) an intrinsic oscillation should develop preferentially in the Eastern Pacific, while in the Central Pacific the flow should be in large part controlled by the features of the random wind disturbances. This, however, assumes that the Eastern and Central Pacific are independent.

There is also the possibility that changes in the leading processes over the equatorial Pacific modify the areas of convective/absolute instability from one El Niño event to another and, consequently, the location of peak SST anomalies. For example, focus has been given recently to a new type of El Niño with peak SST anomalies in the Central Pacific, called 'Modoki' El Niño, 'Warm Pool' or 'Central Pacific' El Niño (Ashok et al., 2007; Kao and Yu, 2009; Yeh et al., 2009; Dewitte et al., 2011). Kug et al. (2009) noted that this type of event is related to stronger zonal advective and atmospheric damping processes, while the conventional 'Cold Tongue' event with peak anomalies in the Eastern Pacific is more related to thermocline processes. For this type of event, the discharge process of the equatorial heat content is not efficient enough to trigger the reversal from the warm to the cold phase, which suggests a secondary role of boundary reflections. This questions to what extent the 'Central Pacific' El Niño may be accounted for by the absolute regime documented in this study.

\section{Acknowledgements}

S. Thual has been supported by CNRS and Conseil Régional Midi-Pyrénées under contract No. 022009. The authors would like to thank Professors Nick Hall, Soon-Il An and Sang-Wook Yeh for fruitful discussions, as well as the two anonymous reviewers.

\section{References}

An S-I, Jin F-F. 2001. Collective role of thermocline and zonal advective feedbacks in the ENSO Mode. J. Climate 14: 3421-3432.

Ashok K, Behera SK, Rao SA, Weng H, Yamagata T. 2007. El Niño Modoki and its possible teleconnection. J. Geophys. Res. 112: C11007, DOI: 10.1029/2006JC003798.

Bejarano L, Jin F-F. 2008. Coexistence of equatorial coupled modes of ENSO. J. Climate 21: 3051-3067.

Briggs R. 1964. Electron-Stream Interaction with Plasmas. MIT Press: Cambridge, MA.

Dewitte B, Choi J, An S-I, Thual S. 2011. Vertical structure variability and equatorial waves during central Pacific and eastern Pacific El Niños in a coupled general circulation model. Clim. Dynam. 38: 2275-2289.

Dommenget D. 2010. The slab ocean El Niño. Geophys. Res. Lett. 37: L20701, DOI: 10.1029/2010GL044888.

Fedorov AV, Philander SG. 2001. A stability analysis of tropical ocean-atmosphere interactions: bridging measurements and theory for El Niño. J. Climate 14: 3086-3101.

Gill AE. 1980. Some simple solutions for heat-induced tropical circulation. Q. J. R. Meteorol. Soc. 106: 447-462.

Harrison DE, Vecchi GA. 1997. Westerly wind events in the tropical Pacific, 1986-95. J. Climate 10: 3131-3156.

Hirst AC. 1986. Unstable and damped equatorial modes in simple coupled ocean-atmosphere models. J. Atmos. Sci. 43: 606-632.

Huerre P, Monkewitz PA. 1990. Local and global instabilities in spatially developing flows. Annu. Rev. Fluid Mech. 22: 473-537.

Jin F-F. 1997. An equatorial ocean recharge paradigm for ENSO. Part I: Conceptual model. J. Atmos. Sci. 54: 811-829.

Jin F-F, Neelin JD, Ghil M. 1994. El Nino on the Devil's Staircase: annual subharmonic steps to chaos. Science 264: 70-72.

Kao H-Y, Yu J-Y. 2009. Contrasting Eastern-Pacific and Central-Pacific types of ENSO. J. Climate 22: 615-632.

Kessler WS. 2002. Is ENSO a cycle or a series of events? Geophys. Res. Lett. 29: 2125, DOI: 10.1029/2002GL015924.

Kleeman R. 2008. Stochastic theories for the irregularity of ENSO. Phil. Trans. R. Soc. A 366: 2509-2524.

Kug J-S, Jin F-F, An S-I. 2009. Two types of El Niño events: Cold Tongue El Niño and Warm Pool El Niño. J. Climate 22: 1499-1515.

Neelin JD. 1991. The slow sea surface temperature mode and the fastwave limit: analytic theory for tropical interannual oscillations and experiments in a hybrid coupled model. J. Atmos. Sci. 48: 584-606. 
Neelin JD, Battisti DS, Hirst AC, Jin F-F, Wakata Y, Yamagata T, Zebiak SE. 1998. ENSO theory. J. Geophys. Res. 103(C7): 14261-14290. Pedlosky J. 2012. Instability theory for the oceans and atmosphere. http://www.whoi.edu/profile.do?id=jpedlosky

Philander SG, Fedorov A. 2003. Is El Niño sporadic or cyclic? Annu. Rev. Earth Planet. Sci. 31: 579-594.

Picaut J, Masia F, du Penhoat Y. 1997. An advective-reflective conceptual model for the oscillatory nature of the ENSO. Science 277: 663-666.

Pontaud M, Thual O. 1995. Some effects of a mean zonal thermocline gradient on planetary equatorial waves. Ann. Geophys. 13: $1223-1228$.

Pontaud M, Thual O. 1998. Coupled process for equatorial Pacific interannual variability. Q. J. R. Meteorol. Soc. 124: 527-555.

Suarez MJ, Schopf PS. 1988. A delayed action oscillator for ENSO. J. Atmos. Sci. 45: 3283-3287.
Suslov SA. 2006. Numerical aspects of searching convective/absolute instability transition. J. Comput. Phys. 212: 188-217.

Thual S, Dewitte B, An S-I, Ayoub N. 2011. Sensitivity of ENSO to stratification in a recharge-discharge conceptual model. J. Climate 24: $4332-4349$.

Timmermann A, Jin F-F, Abshagen J. 2003. A nonlinear theory for El Niño bursting. J. Atmos. Sci. 60: 152-165.

Wang B, Xie X. 1998. Coupled modes of the warm pool climate system. Part I: The role of air-sea interaction in maintaining Madden-Julian oscillation. J. Climate 11: 2116-2135.

Wang C, Weisberg RH. 1996. Stability of equatorial modes in a simplified coupled ocean-atmosphere model. J. Climate 9: 3132-3148.

Weisberg RH, Wang C. 1997. A western Pacific oscillator paradigm for the El Niño-Southern Oscillation. Geophys. Res. Lett. 24: 779-782.

Yeh S-W, Kug J-S, Dewitte B, Kwon M-H, Kirtman BP, Jin F-F. 2009. El Nino in a changing climate. Nature 461: 511-514. 